\title{
$G$-ELLIPTIC SYSTEMS AND THE GENUS ZERO PROBLEM FOR $M_{24}$
}

\author{
GEOFFREY MASON
}

\section{INTRODUCTION}

Toward the end of the 1970s J. H. Conway and S. P. Norton wrote a quite unique and influential paper [3] in which they produced incontrovertible evidence, based partly on ideas of J. McKay and J. G. Thompson [13], that the Fischer-Griess Monster simple group $M$ was intimately related to the modular function $j(\tau)$ familiar from the theory of modular forms. Thus was so-called 'Moonshine' brought into the world. Among other things, it was conjectured that there is a natural infinite-dimensional complex vector space $V^{\sharp}$ which carries a $\mathrm{Z}$-grading into finite-dimensional subspaces $V^{\sharp}=V_{-1} \oplus V_{1} \oplus V_{2} \oplus \cdots$ such that each $V_{i}$ is a $\mathbf{C} M$ module. Moreover the graded character $\operatorname{ch}_{V^{\sharp}}(q):=\sum_{n \geq-1} \operatorname{dim} V_{n} q^{n}$ of $V^{\sharp}$ should be the Fourier expansion of $j(\tau)$, namely $q^{-1}+$ $196884 q+21,493,760 q^{2}+\cdots$ if the indeterminate $q$ is interpreted as $e^{2 \pi i \tau}$ for $\tau$ in the upper half-plane $H$. There should in addition be an analogous interpretation for

$$
\operatorname{tr}_{V^{\sharp}}(g, q):=\sum_{n \geq-1} \operatorname{trace}_{V_{n}}(g) q^{n}
$$

for each $g \in M$; namely, corresponding to $g$ there is a discrete subgroup $\Gamma_{g}$ of $S L_{2}(\mathbf{R})$ of genus zero i.e., the compactified orbit space $\left(\Gamma_{g} \backslash H\right)^{*}$ is a sphere, such that $\operatorname{tr}_{V^{\sharp}}(g, q)$ is a modular function invariant under $\Gamma_{g}$ which generates the field of all such functions over C. (Briefly, $\operatorname{tr}_{V^{\sharp}}(g, q)$ is a so-called 'hauptmodul'.)

This conjecture is known as the genus zero problem for $M$ and has recently been settled in the affirmative. The work of Frenkel-

Received by the editors March 1990 and, in revised form, December 5, 1990.

1980 Mathematics Subject Classification (1985 Revision). Primary 11F22; Secondary $11 \mathrm{~F} 25,20 \mathrm{D} 08,81 \mathrm{R} 05$.

Supported by grants from the NSF; research at MSRI supported in part by NSF Grant DMS-8505550. 
Lepowsky-Meurman [7] and Borcherds [1] established the existence of a $V^{\sharp}$ with the correct graded character and, equally importantly, showed that the proper setting for studying such questions is conformal field theory and the theory of chiral algebras. Very recently, Borcherds [2] has announced a solution of the remainder of the genus zero problem for $M$ by indeed verifying the hauptmodul property of $\operatorname{tr}_{V^{\sharp}}(g, q)$, but it is perhaps fair to say that the true meaning of this property is still something of a mystery.

In the meantime, Norton has considerably extended the genus zero problem. In an appendix to [12], he suggested the possibility of attaching to each element $g \in M$ a certain complex vector space $V_{g}^{\sharp}$ which is graded (by rational numbers with bounded denominator) into finite-dimensional $C_{M}(g)$-modules $\left(C_{M}(g)=\right.$ $\{h \in M \mid g h=\{g\})$, so that $V_{1}^{\sharp}$ is our original space $V^{\sharp}$ and for $h \in C_{M}(g)$ the $q$-expansion $\operatorname{tr}_{V_{g}^{\sharp}}(h, q)$ is either a constant or again a hauptmodul. Furthermore there is a certain 'compatibility' between the various trace functions afforded by the modular group $S L_{2}(\mathbf{Z})$-see below for more details.

This conjecture remains open, but it has been pointed out by physicists (cf. [5, 14] for example) that there is again a 'stringy' perspective from which to understand it: conformal field theory on an orbifold. Very roughly, if a finite group $G$ acts smoothly on a manifold $X$, one considers the space of all smooth (in a suitable sense) maps $S^{1} \rightarrow G \backslash X$. This decomposes into subsets indexed by $g \in G$ corresponding to maps $f:[0,1] \rightarrow X$ such that $f(1)=g f(0)$. After implementation of a suitable quantization procedure, what results is a Hilbert space $H_{g}$ which enjoys all of the properties of $V_{g}^{\sharp}$ sketched above except perhaps the hauptmodul property of the trace functions, which appears to be a relatively unusual occurrence.

Thus this point of view leads to the tantalizing question of whether the Monster $M$ acts on a suitable (24-dimensional?) manifold $X$ in such a way that the extended genus zero problem of Norton can be explained. Furthermore, it suggests developing a moonshine type theory for arbitrary finite groups. Do other of the so-called sporadic groups (cf. [8]) enjoy monstrous properties? The results of this announcement are a step in this direction. We show how, given any finite group $G \leq S O(24 n, \mathbf{R})$, there is attached a certain family $\left\{H_{g}\right\}$ of explicitly described complex vector spaces, with all of the expected properties. Furthermore, 
in at least one case, $M_{24} \leq S O(24, \mathbf{R})$ where $M_{24}$ is the largest sporadic Mathieu group [loc cit.], the extended genus zero property holds. One of the novelties of our approach, however, is that the construction of the family $\left\{H_{g}\right\}$ proceeds not from a group action on a manifold but from a quite different perspective. We adopt the vantage point of classical number theory and following [11] introduce a theory of Hecke-type operators into the study of $G$-elliptic systems (see below), so that for us the modular invariance of the trace functions-a crucial issue in conformal field theory-depends on the theory of these operators.

\section{Statement of Results}

For a finite group $G$, let $P(G)=P=\{(g, h) \in G \times G \mid g h=$ $h g\}$. If $\Gamma=S L_{2}(Z)$ and $\left(\begin{array}{ll}a & b \\ c & d\end{array}\right) \in \Gamma$, define

$$
(g, h) \cdot\left(\begin{array}{ll}
a & b \\
c & d
\end{array}\right)=\left(g^{a} h^{c}, g^{b} h^{d}\right)
$$

Then the map $\alpha:(g, h) \mapsto(g, h) \cdot \alpha^{-1}$ defines a left action of $\Gamma$ on $P$. Let $\mathscr{M}$ be the space of nowhere-vanishing $\mathbf{C}$-valued functions on $P$, which we can naturally regard as a (multiplicative) right $\mathbf{Z} \Gamma$-module.

Let $k: P \rightarrow \mathbf{Z}$ be a $\Gamma$-invariant weight function and $j(\alpha, \tau)=$ $(c \tau+d)$ the usual 1-cocycle of $\Gamma$ associated to its left action on $H$, where $\alpha=\left(\begin{array}{ll}a & b \\ c & d\end{array}\right) \in \Gamma$. Then for a 1-cocycle $\sigma$ in $C^{1}(\Gamma, \mathscr{M})$ there is a right action of $\Gamma$ on functions $f: P \times H \rightarrow \mathbf{C}$ given by

$$
\begin{aligned}
& \left(f \|_{k, \sigma} \alpha\right)(g, h ; \tau) \\
& \quad=\sigma(\alpha ; g, h) j(\alpha, \tau)^{-k(g, h)} f\left((g, h)^{-1} \alpha ; \alpha \tau\right)
\end{aligned}
$$

where in (2.2) we have set $\sigma(\alpha ; g, h)=\sigma(\alpha)((g, h))$.

Definition. A G-elliptic system consists of the following data:

(i) A weight function $k$ and 1-cocycle $\sigma$ as above.

(ii) For each $g \in G$, a (rationally) graded virtual complex vector space

$$
H_{g}=\bigoplus_{n \in \mathbf{Z}} H_{g}^{n} q^{n / D_{g}}
$$

with $H_{g}^{n}$ a virtual $C_{G}(g)$-module, $q$ indeterminate, $D_{g}$ an integer depending only on $g$. Moreover the following 
are required to be satisfied:

If $(g, h) \in P$ and $f(g, h ; \tau):=\sum \operatorname{tr}_{H_{g}^{n}}(h) q^{n / D_{g}}$ is the graded trace of $h$ on $H_{g}, q=e^{2 \pi i \tau}$, then

(iii) $f(g, h ; \tau)$ is meromorphic on $H \cup\{\infty\}$.

(iv) $f \|_{k, \sigma} \alpha=f$ for all $\alpha \in \Gamma$.

(v) $f(g, h ; \tau)=f\left(g^{x}, h^{x}, \tau\right)$ for all $x \in G$.

It is not hard to see that (iii) and (iv) already force $f(g, h ; \tau)$ to be a modular form of weight $k(g, h)$, and a $G$-elliptic system is essentially a collection of modular forms (and their $q$-expansions at the cusps) together with an interpretation as trace functions. A discussion of this can be found in [9]. Orbifolds provide examples where each $H_{g}$ is a genuine representation space, the weight function $k$ is identically zero, and each $\sigma(\alpha ; g, h)$ is a root of unity. Norton's extended genus zero problem for the Monster is that such an elliptic system exists for $M$-together with the hauptmodul property.

We say that the $G$-elliptic system $\left\{H_{g}\right\}$ is of weight zero if the weight function $k$ is identically zero i.e., if each trace function $\operatorname{trace}\left(h\right.$ on $\left.H_{g}\right)$ is a modular function of weight zero. The $G$ elliptic systems we are interested in describing are of this type and depend only on an embedding $G \leq S O(24 d, \mathbf{R}), d$ an integer.

Let $V$ be the underlying orthogonal space with scalars extended to $\mathrm{C}$, and set $W=V \oplus V$.

Fix $g \in G$. Using the shorthand $e(x)$ for $\exp (2 \pi i x)$, let $0<b_{1}<b_{2}<\cdots<b_{r} \leq 1$ be such that $e\left(b_{j}\right)$ are the distinct eigenvalues of $g$ in its action on $V$, and let $V_{j}$ (resp. $W_{j}$ ) be the corresponding eigenspace. We define graded $C_{G}(g)$-modules as follows:

$$
\begin{gathered}
M_{g}=\bigoplus_{j=1}^{r} \bigoplus_{\substack{i=0 \\
*}}^{\infty} W_{j} q^{i+b_{j}-1 / 2} \\
N_{g}=\bigoplus_{j=1}^{r} \bigoplus_{i=0}^{\infty} W_{j} q^{i+b_{j}}
\end{gathered}
$$

and a linear (degree 1 ) character given by

$$
\lambda_{g}(h)=\operatorname{det} \cdot h^{-1} \text { on } \bigoplus_{b_{j}>1 / 2} V_{j}, \quad h \in C_{G}(g) .
$$

In $(2.3), *$ indicates that the sum runs only over indices satisfying $i+b_{j}>1 / 2$. 
As $W_{1}$ and $W_{1 / 2}$ have a structure of real orthogonal spaces admitting the diagonal action of $C_{G}(g)$, we may consider the usual $1 / 2$-spin modules $\Delta_{g}^{ \pm}$for the corresponding real spin group affording representations of $C_{G}(g)$. Similarly $\Delta_{g}$ is the restriction of the spin-module for the real spin group corresponding to $W_{1}$.

Next, let $B_{2}(X)=X^{2}-X+1 / 6$ denote the second Bernoulli polynomial, and set

$$
B(g)=\frac{1}{4} \sum_{j=1}^{r} \operatorname{dim} V_{j} B_{2}\left(b_{j}\right) .
$$

For a (graded) module $M$ let $\Lambda^{ \pm}(M)$ denote the even and odd parts of the exterior algebra on $M$, that is $\Lambda^{+}(M)=\bigoplus_{k \geq 0} \Lambda^{2 k}(M)$, $\Lambda^{-}(M)=\bigoplus_{k \geq 0} \Lambda^{2 k+1}(M)$.

Finally, if $S=\left(\begin{array}{cc}0 & -1 \\ 1 & 0\end{array}\right), T=\left(\begin{array}{ll}1 & 1 \\ 0 & 1\end{array}\right)$ are the standard generators of $\Gamma$ and $h \in C_{G}(g)$, define

$$
\sigma(T ; g, h)=e\left\{\sum_{\substack{j \\ b_{j}<1 / 2}} b_{j}^{2} \operatorname{dim} V_{j}-\frac{1}{8} \operatorname{dim} V_{1 / 2}\right\}
$$

$$
\sigma(S ; g, h)=e\left\{-2 \sum_{\substack{j \\ b_{j}<1 / 2}} \sum_{i=1}^{\operatorname{dim} V_{j}} b_{j} b_{j_{i}}^{\prime}-\frac{1}{4} \operatorname{dim} V_{1 / 2,1 / 2}\right\}
$$

where in (2.8), $e\left(b_{j_{1}}^{\prime}\right), e\left(b_{j_{2}}^{\prime}\right), \ldots$ runs over the eigenvalues of $h$ (with multiplicity) on $V_{j}$ and $-1 / 2<b_{j_{i}} \leq 1 / 2$, and $V_{1 / 2,1 / 2}$ is the subspace of $V$ on which both $g$ and $h$ act as -1 .

Remark. We have $\sigma(S ; g, g)^{-1}=\sigma(T ; g, h)^{2}$. For the relevance of this to orbifold theory, see [4].

Theorem 1. The function $\sigma$ defined by (2.7) and (2.8) extends to a 1-cocycle $\sigma \in C^{1}(\Gamma, \mathscr{M})$.

Theorem 2. For $g \in G$, set

$$
\begin{aligned}
H_{g}= & q^{B\left(g^{2}\right)-2 B(g)}\left(\Delta_{g}^{+} \otimes \Lambda^{+}\left(M_{g}\right) \oplus \Delta_{g}^{-} \otimes \Lambda^{-}\left(M_{g}\right)\right) \\
& +1 / 2 q^{2 B(g)} \lambda_{g} \otimes \Delta_{g} \otimes \Lambda\left(N_{g}\right) .
\end{aligned}
$$


Then $\left\{H_{g}\right\}$ is an elliptic system of weight zero corresponding to the 1 -cocycle $\sigma$ of $\Gamma$ given by Proposition 1 .

Remarks. (1) There is a 'denominator' of 2 in (2.9). This can be removed, yielding a true representation space for $C_{G}(g)$ if, for example $\Delta_{g}$ is the sum of a pair of isomorphic $C_{G}(g)$-modules. This in turn holds, for example, if $G$ fixes a nonzero vector in $V$; in particular, this is the case in Theorem 3.

(2) The character of $H_{1}$ i.e. $f(1,1, \tau)$ is a monic polynomial in $j(\tau)$ of degree $d$ (recall $\operatorname{dim} V=24 d$ ). More precisely, we have

$$
f(1,1, \tau)=\operatorname{char} H_{1}=T_{2} \Delta^{d} / \Delta^{d}
$$

where $\Delta=q \prod_{n=1}^{\infty}\left(1-q^{n}\right)^{24}$ is the discriminant and $T_{2}$ is the second Hecke operator. There is an analogous, but more complicated, identity for the other trace functions.

Theorem 3. Take $G=M_{24} \leq S O(24, \mathbf{R})$ and let $\left\{H_{g}\right\}$ be as in Theorem 2. Then this elliptic system has the genus zero property. Precisely, if $(g, h) \in P\left(M_{24}\right)$ and $f(g, h ; \tau)=\operatorname{trace}\left(h\right.$ on $\left.H_{g}\right)$ then one of the following holds:

(a) $\langle g, h\rangle$ has noncyclic Sylow 2-subgroup and $f(g, h ; \tau)$ $=0$.

(b) $\langle g, h\rangle$ has cyclic Sylow 2-subgroup, and if $\Gamma_{g, h}$ is the full invariance group of $f(g, h ; \tau)$ is $S L_{2}(\mathbf{R})$ then $\left(\Gamma_{g, h} \backslash H\right)^{*}$ is a sphere and the function field is generated by $f(g, h ; \tau)$.

\section{SKETCH OF PROOFS}

Keeping earlier notation, set

$$
L_{g}=q^{B(g)} \Lambda\left(-\bigoplus_{j=1}^{r} \bigoplus_{n=0}^{\infty} V_{j} q^{n+b_{j}}\right) .
$$

Lemma 1. Assume that $G$ acts on $V$ evenly i.e., for each $(g, h) \in$ $P$ we have $\operatorname{dim} C_{V}(\langle g, h\rangle)$ even. Then $\left\{L_{g}\right\}$ is a (virtual) elliptic system with respect to a certain $\beta \in C^{1}(\Gamma, \mathscr{M})$ and weight function $k:\langle g, h\rangle \mapsto 1 / 2 \operatorname{dim} C_{V}(\langle g, h\rangle)$. (A discussion of a special case is already contained in [10].)

We wish to define a theory of Hecke-type operators which will act on $\left\{L_{g}\right\}$ and produce a second elliptic system for us. This 
entails extending (2.2) to actions of $\alpha \in G L_{2}(\mathbf{Z})$. There is a left action of $G L_{2}(\mathbf{Z})$ extending (2.1), namely

$$
\alpha:(g, h) \mapsto(g, h) \alpha^{\prime}, \alpha^{\prime}=(\operatorname{det} \alpha) \alpha^{-1}
$$

but to extend (2.2) we must introduce an index in the fashion of Jacobi forms (cf. [6]). Precisely, the 1-cocycle $\beta$ of Lemma 1 factors as $\gamma \delta$ where $\gamma \in C^{1}(\Gamma, \mathscr{M})$ and $\delta$ is a twisted 1-cocycle in the sense that

$$
\delta\left(\alpha_{1} \alpha_{2} ; g, h\right)=\delta\left(\alpha_{1} ;(g, h) \alpha_{2}^{\prime}\right) \delta\left(\alpha_{2} ; g, h\right)^{\operatorname{det} \alpha_{1}}
$$

for $\alpha_{1}, \alpha_{2} \in G L_{2}(\mathbf{Z})$. Note that $\delta(\alpha ; g, h)$ may well vanish, but not if $\operatorname{det} \alpha=1$. Now define for $f: P \times H \rightarrow \mathbf{C}$,

$$
\begin{aligned}
f \|_{k, m, \gamma, \delta} \alpha(g, h ; \tau)= & (\operatorname{det} \alpha)^{1 / 2 k(g, h)} j(\alpha, \tau)^{-k(g, h)} \\
& \times f\left((g, h) \alpha^{\prime} ; \alpha \tau\right) \gamma(\alpha ; g, h) \delta(\alpha ; g, h)^{m} .
\end{aligned}
$$

We have

$$
f_{k, m, \gamma, \delta}\left\|\alpha \beta=\left(f \|_{k, m, \gamma, \delta} \alpha\right)\right\|_{k, m \operatorname{det} \alpha, \gamma, \delta} \beta
$$

in case either (i) $\operatorname{det} \alpha \beta$ is odd or (ii) $V$ is the sum of a pair of isomorphic $\mathrm{C} G$-modules (e.g., replace $V$ by $W$ ). Under either of these conditions we can develop a theory of Hecke operators. To some extent this is formal: for a double coset $\Gamma \alpha \Gamma$ of $\Gamma$ in $G L_{2}(\mathbf{Z})$ define

$$
\begin{aligned}
& f \|_{k, m, \gamma, \delta}[\Gamma \alpha \Gamma](g, h ; \tau) \\
& \quad=(\operatorname{det} \alpha)^{k(g, h) / 2-1} \sum_{i} f \|_{k, m, \gamma, \delta} \alpha_{i}(g, h ; \tau)
\end{aligned}
$$

where $\alpha_{i}$ runs over representatives of $\Gamma \backslash \Gamma \alpha \Gamma$, which is well defined by (3.5). According as cases (i) or (ii) above take $n$ odd or arbitrary and set

$$
T_{n} f=\sum f \|_{k, m, \gamma, \delta}[\Gamma \alpha \Gamma]
$$

with the sum running over all double cosets of $\Gamma$ in the matrices of determinant $n$ in $G L_{2}(Z)$. Then by (3.5) we get

$$
T_{n} f \|_{k, n, \gamma, \delta} \alpha=T_{n} f, \quad \alpha \in \Gamma
$$

if $f$ is the trace function of the elliptic system $\left\{L_{g}\right\}$ of Lemma

1. The hard part is to now establish 
Proposition 2. There is a G-elliptic system $\left\{E_{g}\right\}$ and a function $\varepsilon: P(G) \rightarrow\{$ roots of unity $\}$ with the following properties:

(a) The weight function is the same as that for $\left\{L_{g}\right\}$.

(b) The 1-cocycle $\gamma^{\prime} \in C(\Gamma, \mathscr{M})$ associated with $\left\{E_{g}\right\}$ is given by $\beta^{\prime}=\gamma_{\varepsilon} \gamma \delta^{n}$ where $\gamma_{\varepsilon}$ is the 1-coboundary determined by $\varepsilon$.

(c) The trace function $F(g, h ; \tau)$ of $\left\{E_{g}\right\}$ satisfies $F=$ $\varepsilon^{-1} T_{n} f$.

Theorem 2 follows from the case $n=2$ by taking the quotient elliptic system $\left\{E_{g}\right\} /\left\{L_{g}\right\}$ (note: the denominator never vanishes!) while the 1-cocycle $\sigma$ of Theorem 1 is just $\gamma_{\varepsilon} \delta$. Because we have complete control over the various 1-cocycles, i.e. they are computable in terms of the action of $G$ on $V$, the functional equation (3.8) for $T_{2} f$ and the analogue for $f$ itself lead to the proof of Theorem 3 .

\section{REFERENCES}

1. R. Borcherds, Vertex algebras, Kac-Moody algebras and the Monster, Proc. Nat. Acad. Sci. 83 (1986), 3068-3071.

2. __ preprint, Cambs. Univ. DPMMS (1990).

3. J. Conway and S. Norton, Monstrous moonshine, Bull. London Math. Soc. 11 (1979), 303-339.

4. R. Dijkgraaf, C. Vafa, E. Verlinde, and H. Verlinde, The operator algebra of orbifold models, Comm. Math. Phys. 123 (1989), 485-526.

5. L. Dixon, P. Ginsparg, and J. Harvey, Beauty and the Beast: Superconformal symmetry in a Monster module, Comm. Math. Phys. 119 (1988), 221-241.

6. M. Eichler and D. Zagier, Introduction to Jacobi Forms, Birkhäuser, Boston, 1986.

7. I. Frenkel, J. Lepowsky, and A. Meurman, Vertex operator algebras and the Monster, Academic Press, New York, 1988.

8. D. Gorenstein, Finite simple groups, Plenum, New York, 1985.

9. G. Mason, Remarks on moonshine and orbifolds, Proc. London Math. Soc. Conf., Durham (1990) (to appear).

10. __ Elliptic systems and the eta-function, Notas Soc. Mat. Chile 8 (1989), 37-53.

11. __ Finite groups and Hecke operators, Math. Ann. 283 (1989), 381-409.

12. _. Finite groups and modular functions, Proc. Sympos. Pure Math., vol. 47, Amer. Math. Soc., Providence, RI, 1987. 
13. J. Thompson, Finite groups and modular functions, Bull. London Math. Soc. 11 (1979).

14. M. Tuite, Monstrous moonshine from orbifolds, preprint, Dublin Inst. for Adv. Studies. (1990).

Mathematical Sciences Research Institute, Berkeley, California

Current address: Department of Mathematics, University of California at Santa Cruz, Santa Cruz, California 95064 
